\title{
A New Method for Judging the Relevance of Combat Capabilities of Weapon System
}

\author{
Xinhua He, Weichao Zhang, Hao Cheng and Qiang Qu \\ Department of Information and Communication, Beijing, China
}

\begin{abstract}
The evaluation and analysis of the combat capability of the weapon and equipment system is one of the hot issues to be studied in the contemporary military development process. Since it is a complex system, the correlation between the internal components has a greater impact on the assessment of combat capability. Therefore, how to accurately The ability to evaluate it is a problem that needs urgent solution. In order to solve this problem, this paper proposes a partial least squares analysis method based on multiple variables, and concretely analyses the association between the various components in a complex system. The feasibility of this method is verified by concrete examples.
\end{abstract}

Keywords-weaponry combat capability; connection relation; multiple variables; pls

\section{INTRODUCTION}

The analysis and demonstration of the weapon and equipment system are the core elements of the current military development. Therefore, assessing the combat capability of the weaponry and equipment system accurately and effectively is the current research focus. Due to the complex relationship between the various weapon systems which makes up the entire system, such an association is the key to the emergence of the overall combat capability of the weapon and equipment system. So the determination of this relationship is of the particular importance in the assessment of the combat capability of the weapons and equipment system.

In the course of evaluating the combat capability of the weapon and equipment system, most of the judgments about the relation within the system are based on the expert evaluation method. Due to its subjectivity and restraint, so the judgment of the association has a greater impact because of the lack of scientific basis. Therefore, this paper presents a decision method based on multivariate partial least-squares correlation, in order to avoid the assessment process overreliance on experts and the impact of the assessment results, and make up for the lack of traditional assessment methods.

\section{AN OVERVIEW OF PARTIAL LEAST SQUARES}

Partial Least square was proposed by Swedish Scientists S. Wold and C. Albano in order to solve the related problems between the variables in the metrological chemistry. It is a data analysis method based on multiple variables statistics [1].

Partial least squares establish the description of the correlation between the data blocks by finding the sum of the squares of the minimum error between the data [2]. It is a multi-dependent variable on the multi-independent variables regression method, and it is more suitable to analyze the existence of high correlation between variables, removes the extraneous useless information in the information and the interference caused by non-explanatory information through the sample data information sub-screening, this can be more effective in solving the multiple correlations between variables [3] and achieve the data structure simplification at the same time the model was sat up [4]. Therefore, this method of data analysis is suitable for describing the relationship between the data in the process of analyzing the operational capability of weapon system.

\section{Multivariate Partial Least Souares Modeling}

Traditional partial least squares analyze the correlation between independent variable and dependent variable. However, this correlation analysis cannot meet the needs of complex weapon system evaluation of combat capability. Therefore, this paper proposes a method based on multivariate partial least squares to analyze the correlation between each weapon system.

The structural equation modelling process of PLS based on multiple variables is similar to the traditional PLS modelling process, which is mainly divided into four main steps: model setting, model identification, model estimation and model evaluation [5]. The model setting is to establish the causal relationship between variables and indicators and set the hypothesis of the initial theoretical model; The model identification is to assign the only estimate of each free parameter in the system to the model and test the deviation convergence of the model. The model estimation is the key step in the modelling process and has a decisive influence on the estimated value, the error value and the fitting degree of the variables. The model evaluation is to evaluate the proposed model to determine whether it can fully explain the analysis of the data [6].

\section{A. Data Preprocessing}

Data preprocessing refers to some optimization of the data before the PLS regression calculation, for example, the audit of abnormal data screening and missing data to fill such operations. This article uses the statistical products and services solutions software SPSS to solve the data exception handling and missing processing issues [7-8]. In addition, the underlying data can be obtained through a large number of actual combat 
drills and simulation simulations, and the data needs to be normalized so as to give full play to their value.

\section{B. Model Setting}

Set that there are a total of $m$ group significant variables, each group of significant variables contains $n$ observation variables, expressed as $A_{i}=\left\{a_{i 1}, a_{i 2} \ldots a_{i n}\right\}$, $a_{i j}[i \in(1, m) ; j \in(1, n)]$ is any observation variable in $A_{i}$, the latent variable corresponding to each group $A_{i}$ is $\xi_{i}(i=1,2 \ldots m)$. The specific model is as follows [9-10]:

- Measurement mode

$$
a_{i j}=\Gamma_{i j} \xi_{1}+\kappa_{i j}
$$

Where $\Gamma_{i j}$ is the load index of the indicator $a_{i j}$, and $\kappa_{i j}$ is the residual. The above model is assumed in the partial least squares algorithm to satisfy the following relationship:

- $\quad$ Desired relationship:

$$
E\left(a_{i j} \mid \xi_{i}\right)=\Gamma_{i j} \xi_{i}
$$

- $\quad$ Latent variable variance unit:

$$
\operatorname{Var}\left(\xi_{i}\right)=1
$$

- Non-relatedness:

$$
\begin{gathered}
\operatorname{cov}\left(\kappa_{i j}, \xi_{i}\right)=r\left(\kappa_{i j}, \xi_{i}\right)=0 \\
\operatorname{cov}\left(\kappa_{i j}, \kappa_{i j}\right)=r\left(\kappa_{i j}, \kappa_{i j}\right)=0(i \neq j)
\end{gathered}
$$

- Structural model

$$
\xi_{i}=\sum_{i=0}^{m} \alpha_{i j} \xi_{i}+\sigma_{i}
$$

Where $\sigma_{i}$ is the random error, different modes are chosen for different types of latent variables. Mode A is selected as endogenous latent variable and mode B is used as exogenous latent variable.

\section{Model Recognition}

In this paper, the IBM SPSS Statistics data editor is used to verify the recognition. SPSS calculates the Kolombach's coefficient of the model data. The result is used as an indicator of reliability, as long as the reliability coefficient is greater than 0.7. The specific formula is as follows [10]:

$$
\alpha=\frac{k}{k-1}\left(1-\sum \frac{S_{i}^{2}}{S_{t}^{2}}\right)
$$

$$
S_{i}^{2}=\frac{\sum a_{t}^{2}}{n}-\left(\frac{\sum a_{t}^{2}}{n}\right)^{2}
$$

$$
\sum_{1}^{k} S_{i}{ }^{2}=\sum_{1}^{k} \frac{\sum a^{2}}{n}-\sum_{1}^{k}\left(\frac{\sum a}{n}\right)^{2}
$$

Where $\alpha$ is the Kolombach's coefficient of confidence, $k$ is the number of variables.

\section{Model Estimation}

According to the model definition above, the structural equation model is estimated as follows:

Provisions:

$$
\Delta_{i}=f_{i} \sum_{i=1}^{n} \omega_{i j} a_{i j}
$$

$$
f_{i}= \pm\left\{\frac{1}{N} \sum_{n}\left[\sum_{n}\left(\omega_{i j} a_{i j}\right)\right]^{2}\right\}^{\frac{1}{2}}
$$

The weights chosen are as follows:

$$
\text { Mode A: } a_{i j}=\omega_{i j} \Upsilon_{i}+\sigma_{i}
$$

$$
\text { Mode B: } \Upsilon_{i}=\sum\left(\omega_{i j} a_{i j}\right)+\sigma_{i}
$$

Where $\Upsilon_{i}$ is the symbol weight and it is defined as: In the case of multiple latent variables, it is the basis for choosing the weight relationship between the various variables in the model, the formula is as follows: 


$$
\begin{gathered}
\Upsilon_{i}=\sum\left(\gamma_{i j} \Delta_{i}\right) \\
\gamma_{i j}=\operatorname{sign} r\left(\Delta_{i}, \Delta_{j}\right) \begin{cases}1 & r\left(\Delta_{i}, \Delta_{j}\right)>0 \\
0 & r\left(\Delta_{i}, \Delta_{j}\right)=0 \\
-1 & r\left(\Delta_{i}, \Delta_{j}\right)<0\end{cases}
\end{gathered}
$$

The specific steps of the algorithm are as follows:

Step 1: Initialization, set the initial value of the weight value of $\omega_{1}^{(1)}$.

Step 2: Calculate $f_{1}$.

Step 3: Calculate $\Delta_{1}^{(1)}$.

Step 4: Calculate $\Upsilon_{1}^{(1)}$

Step 5: Regression calculation $\omega_{1}^{(2)}$.

Step 6: Judge the relationship between $\omega_{1}^{(1)}$ and $\omega_{1}^{(2)}:\left|\omega_{i}^{(a)}-\omega_{i}^{(a+1)}\right| \leq 10^{-5}$, if the above relationship is established, then proceed to the step 7; else, jump to the step 2.

Step 7: Calculate $\Delta_{i}$.

Step 8: Substitute $\Delta_{i}$ into the corresponding regression equation of the index and latent variables in the regression equation to calculate the parameters for solving each model.

\section{E. Model Evaluation}

After obtaining the estimated value of the latent variable of the model, it is also necessary to perform an overall fitting analysis based on the sample data to determine whether it converges or whether the expected expectation is met or not. This paper evaluates the model by calculating the overall fitting index, such as the fit test, the goodness-of-fit index GFI, the adjusted goodness-of-fit index AGFI, the root mean square error RMESA of the approximate error, and so on [11].

$\chi^{2}$ fitting test which is calculated as:

$$
\chi^{2}=\sum_{i=1}^{k} \frac{\left(f_{i}-n p_{i}\right)^{2}}{n p_{i}}
$$

When $p>0.05$, it means that the fitting is successful.

\section{CASE ANALYSIS}

The above chapters mainly elaborated the theoretical basis of the method of judging the association between multiple variables based on partial least squares. Then this method is applied to the determination of the correlation within the electronic warfare system. Specific operations are as follows:

Smart PLS 2.0 as the platform, the IBM SPSS Statistics data editor is used for data processing, taking the tactical information distribution ability of the equipment system as an example, to show the relationship between each variable. The main process is as follows.

\section{A. Establish an Electronic Countermeasure Capability Index System}

As it shown in Figure 1.

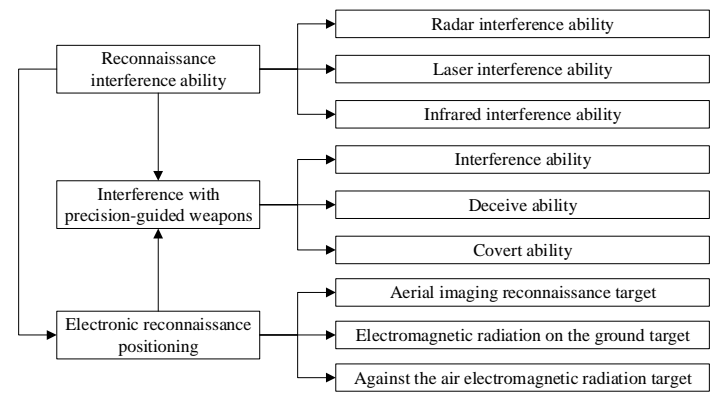

FIGURE I. TACTICAL INFORMATION DISTRIBUTION CAPACITY INDEX SYSTEM DIAGRAM

\section{B. Establish a Path of Electronic Countermeasures.}

The easiest way to model the structural equation model based on Multivariate Partial Least Squares is to describe the correlation between variables and indicators in the model through the path map method [7]. As follows in figure 2:

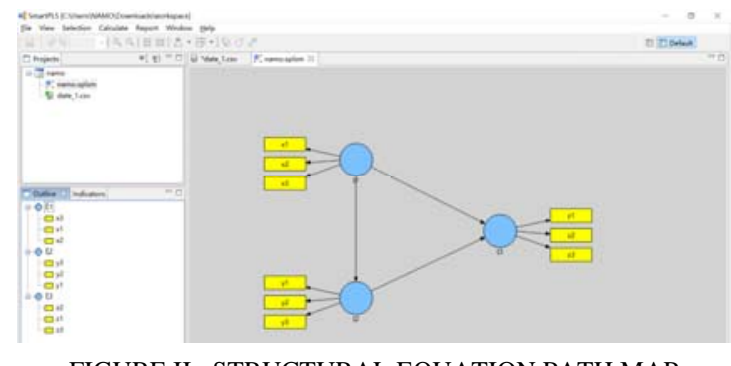

FIGURE II. STRUCTURAL EQUATION PATH MAP

Where $\xi_{1}, \xi_{2}, \xi_{3}$ is a latent variable, $x_{1}, x_{2}, x_{3}$ is the index of $\xi_{1}, y_{1}, y_{2}, y_{3}$ is the index of $\xi_{2}, z_{1}, z_{2}, z_{3}$ is the index of $\xi_{3}$. The observed variables corresponding to each latent variable in Figure 2 are shown in Table 1. 
TABLE I. VARIABLE NAME TABLE

\begin{tabular}{|c|c|c|}
\hline Latent variables & \multicolumn{2}{|c|}{ Significant variables } \\
\hline \multirow[t]{3}{*}{$\xi_{1}$} & $\mathrm{x}_{1}$ & $\begin{array}{l}\text { Radar interference } \\
\text { ability } \\
\end{array}$ \\
\hline & $\mathrm{X}_{2}$ & $\begin{array}{l}\text { Laser interference } \\
\text { ability }\end{array}$ \\
\hline & $\mathrm{x}_{3}$ & $\begin{array}{c}\text { Infrared interference } \\
\text { ability }\end{array}$ \\
\hline \multirow{3}{*}{$\xi_{2}$} & $\mathrm{y}_{1}$ & Interference ability \\
\hline & $\mathrm{y}_{2}$ & Deceive ability \\
\hline & $\mathrm{y}_{3}$ & Covert ability \\
\hline \multirow[t]{3}{*}{$\xi_{3}$} & $\mathrm{Z}_{1}$ & $\begin{array}{l}\text { Aerial imaging } \\
\text { reconnaissance target }\end{array}$ \\
\hline & $\mathrm{z}_{2}$ & $\begin{array}{l}\text { Electromagnetic } \\
\text { radiation on the } \\
\text { ground target }\end{array}$ \\
\hline & $\mathrm{z}_{3}$ & $\begin{array}{l}\text { Against the air } \\
\text { electromagnetic } \\
\text { radiation target }\end{array}$ \\
\hline
\end{tabular}

\section{Data Inputting}

Enter the date into the software through the index values of the electronic warfare system obtained by a military digital simulation experiment center, as shown in figure 3.

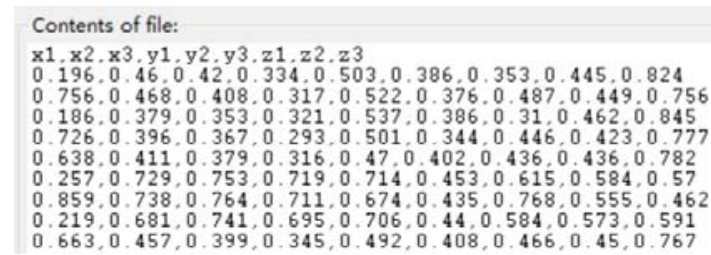

FIGURE III. THE VALUE OF COMBAT CAPABILITY DATA ENTRY CHART

\section{Data Inputting}

Run and the result is shown in Figure 4.

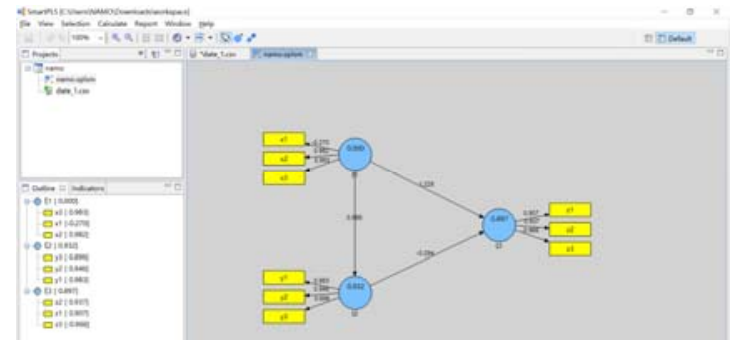

FIGURE IV. RUN RESULTS INTERFACE

\section{E. Model Evaluation and Revision}

Using the above-mentioned model evaluation method to evaluate the model, finally get the correlation between the various indicators of the electronic warfare capability platform.

Then through expert assessment and military research, the analysis of the relationship between the electronic warfare system in line with the actual situation.

\section{CONCLUSIONS}

It is the key to establish a reasonable simulation model that the accurate determination of the relationship between the various constituent units in the simulation of combat capability of the weapon and equipment system. This paper introduces and analyses the multivariate partial least-squares method, and tells about the method to determine the association between multivariate partial least squares. Taking the electronic warfare platform in the weaponry and equipment system as an example, this paper proves the feasibility of the method, this played a certain reference role in the evaluation of the relationship between operational capabilities.

\section{REFERENCES}

[1] Karol Firek,Janusz Rusek. Partial Least Squares Method in the Analysis of the Intensity of Damage in Prefabricated Large-Block Building Structures[J]. Archives of Mining Sciences,2017,62(2).

[2] Qu Haibin. Modelling method based on PLS [J]. Journal of Zhejiang University (Engineering Science), 1999, (05): 19-22

[3] L. Brancheriau,H. Baillères. Use of the Partial Least Squares Method with Acoustic Vibration Spectra as a New Grading Technique for Structural Timber[J]. Holzforschung,2003,57(6).

[4] Karunanidhi Lakshmi,Sivasubramanian Lakshmi. Simultaneous spectrophotometric determination of valsartan and hydrochlorothiazide by H-point standard addition method and partial least squares regression[J]. Acta Pharmaceutica,2011,61(1).

[5] Jahanbakhsh Ghasemi,Hamid R. Seraji,Mohammad Noroozi,Majid Hashemi,Ali Jabbari. Differential Kinetic Spectrophotometric Determinations of Ascorbic Acid and L - Cysteine by Partial Least Squares Method[J]. Analytical Letters,2004,37(4).

[6] Wang Huiwen. Partial least-squares regression method and its application [C]. Beijing: National Defense Industry Press, 1999.4

[7] Lv Zhongkai. PLS-SEM modeling with multiple latent variables [D]. Xinjiang University, 2011.

[8] Hu Jianwen. Exploratory Analysis and Design of Weaponry and Equipment Capability Indicators [M] .Beijing: China Defense Industry Press, 2009: 14-17.

[9] He Tao. PLS algorithm for structural equation model [D]. Tianjin University, 2006.

[10] "SPSS80 Statistical Software Application Guide" published [J]. China Health Statistics, 2000, (03): 33.

[11] WANG Chang-wei, WANG Da-peng, ZHAO Xiao-wen, FANG Qingwei, LIU Yan.Application and Comparison of Fitting Index in Structura Equation Modeling [J]. Modern Preventive Medicine, 2010,37 (01): 7-9. 